\title{
A NOTE ON THE UNIQUENESS OF DOUBLE TRIGONOMETRIC SERIES
}

VICTOR L. SHAPIRO

1. Introduction. Let

$$
\sum a_{M} e^{i M X}
$$

be a double trigonometric series where $a_{M}$ are arbitrary complex numbers and where $M=(m, n), X=(x, y), M X=m x+n y$, and $|M|$ $=\left(m^{2}+n^{2}\right)^{1 / 2}$. The series will be said to converge circularly at point $X$ to $L(X)$ if the circular partial sums of rank $R$,

$$
S_{R}(X)=\sum_{|M| \leqq R} a_{M} e^{i M X},
$$

converge to the finite value $L(X)$. The series will be said to be $(C, 1)$ circularly summable to $L(X)$ if the $(C, 1)$ circular means of rank $R$,

$$
\begin{aligned}
\sigma_{R}(X) & =\frac{2}{R^{2}} \int_{0}^{R} S_{u}(X) u d u=\sum_{|M| \leqq R} a_{M} e^{i M X}\left(1-\frac{|M|^{2}}{R^{2}}\right) \\
& =\sum_{r=0}^{\left[R^{2}\right]}\left(1-\frac{r}{R^{2}}\right) \sum_{|M|^{2}=r} a_{M} e^{i M X},
\end{aligned}
$$

converge to the finite value $L(X)$.

We shall say that (1) is a series of type (U) if

$$
\sum_{1 \leqq|M| \leqq R}\left(a_{M} /|M| 2\right) e^{i M X}
$$

converges uniformly.

It is known, Cheng [1], that if $(1)$ is $(C, 1)$ circularly summable to zero everywhere and is of type (U), then (1) vanishes identically. A result of this nature has for its motivation the theorems of Verblunsky and Cantor in single trigonometric series which obtain uniqueness results without utilizing the notion of type (U), see Zygmund [5, pp. 274 and 297].

2. Statement of main result. It is the purpose of this paper to weaken the hypothesis regarding type (U) in Cheng's result and consequently to obtain a theorem in double trigonometric series which comes a little closer to Verblunsky's. In particular we shall prove the following theorem:

Theorem. Given a double trigonometric series $\sum a_{M} e^{i M X}$ where

Received by the editors January 23, 1953. 
$a_{M}=o\left(|M|^{1-c}\right), \epsilon>0$, are arbitrary complex numbers. Let $E$ be a denumerable closed plane set. Suppose

(i) The series is $(C, 1)$ circularly summable to zero everywhere.

(ii) $F(X)=a_{0}(x+y)^{2} / 4-\lim _{R \rightarrow \infty} \sum_{1 \leq|M| \leqq R}\left(a_{M} /|M|^{2}\right) e^{i M X}$ is continuous on the complement of $E$.

Then the series vanishes identically.

3. Definitions and notations. The open disc of radius $t$ with center $X$ will be designated by $N(X, t)$, and the closed disc by $\bar{N}(X, t)$.

The mean value of $F(X)$ in this disc will be designated by $F_{1, x}(t)$, thus

$$
F_{1, X}(t)=\frac{1}{\pi t^{2}} \int_{N(X, t)} F(P) d P .
$$

$F(X)$ is harmonic in $\bar{N}\left(X_{0}, t_{0}\right)$ will mean there is an $\epsilon>0$ such that $F(X)$ is harmonic in $N\left(X_{0}, t_{0}+\epsilon\right)$.

$J_{k}(u)$ will denote the Bessel function of the first kind and order $k$. $a_{M}=o\left(|M|^{\alpha}\right)$ will mean the following: given an $\epsilon>0$ there exists an $R(\epsilon)$ such that if $|M|>R$, then $\left|a_{M}\right|<\epsilon|M|^{\alpha}$.

4. Fundamental lemmas. In order to prove the theorem we find it necessary to first prove some facts which we shall state as lemmas. The first fact necessary to prove is the existence of the limit in hypothesis (ii) of the theorem. We do so with the following lemma:

LEMMA 1. Given a double trigonometric series $\sum a_{M} e^{i M X}$ where the $a_{M}$ are arbitrary complex numbers. Suppose the series is $(C, 1)$ circularly summable to the finite value $L$ at the point $X_{0}$. Then the series $\sum_{|M| \neq 0}\left(a_{M} /|M|^{2}\right) e^{i M X}$ converges circularly at the point $X_{0}$.

Without loss of generality, we assume that $a_{0}=0$ and $X_{0}=0$. Then setting $A_{i}=\sum_{|M|^{2}-i} a_{M}$, we have by (3) that the single series $\sum_{i=1}^{\infty} A_{i}$ is summable $(C, 1)$ to zero, and consequently that $\sum_{|M| \leqq R} a_{M} /|M|^{2}=\sum_{i=1}^{\left[R^{2}\right]} A_{i} / i$ is convergent.

Lemma 1 tells us that by hypothesis (i), $F(X)$ in hypothesis (ii) is a well-defined function for every point in the plane. The Riesz-Fischer theorem tells us $F(X)$ is in $L_{2}$ over any bounded domain, and consequently for any $N(X, t), F_{1, X}(t)$ exists. We now prove the following lemma concerning this mean:

LEMMA 2. Under the hypotheses of the theorem

$$
F_{1, X}(t)=\frac{a_{0}}{4}\left[(x+y)^{2}+\frac{t^{2}}{2}\right]-2 \sum_{1 \leq|M|} a_{M} e^{i M x} \frac{J_{1}(|M| t)}{|M|^{3} t}
$$


at all points $X$.

Setting $T_{R}(X)=a_{0}(x+y)^{2} / 4-\sum_{1 \leqq|M| \leqq R}\left(a_{M} /|M|^{2}\right) e^{i M X}$, we observe that by the Riesz-Fischer theorem

$$
T_{R}(P) \rightarrow F(P) \quad \text { in } \quad L_{2} \text { on } N(X, t)
$$

and consequently that

$$
\frac{1}{\pi t^{2}} \int_{N(X, t)} T_{R}(P) d P \rightarrow F_{1, X}(t) \quad \text { as } R \rightarrow \infty .
$$

Since $(1 / 2 \pi) \int_{0}^{2 \pi} e^{i t(m \cos \theta+n \sin \theta)} d \theta=J_{0}(|M| t)$ and $\int_{0}^{|M| z} u J_{0}(u) d u=$ $|M| t J_{1}(|M| t)$, we see that the left side of $(4)$ is

$$
\frac{a_{0}}{4}\left[(x+y)^{2}+\frac{t^{2}}{2}\right]-2 \sum_{1 \leqq|M| \leqq R} a_{M} e^{i M X} \frac{J_{1}(|M| t)}{|M|^{3} t},
$$

which fact when combined with (4) proves the lemma.

LEMMA 3. Under the hypothesis of Theorem 1,

$$
F_{1, x}(t)=F(X)+o\left(t^{2}\right)
$$

at all points $X$.

For by Lemmas 1 and 2

$$
\begin{aligned}
\frac{8}{t^{2}}\left[F_{1, I}(t)\right. & -F(X)] \\
& =a_{0}+\lim _{R \rightarrow \infty} \sum_{1 \leq|M| \leqq R} a_{M} e^{i M X} \frac{8}{|M|^{2} t^{2}}\left(1-\frac{2 J_{1}(|M| t)}{|M| t}\right)
\end{aligned}
$$

and the lemma follows from Cheng [1, Theorem 1, p. 404].

We now prove a lemma concerning harmonic functions.

Lemma 4. Let $g(X)$, defined and in $L_{2}$ on $\bar{N}\left(X_{0}, t_{0}\right)$, be harmonic in $\bar{N}\left(X_{0}, t_{0}\right)-X_{0}$. Furthermore, suppose $g_{1, x_{0}}(t)=g\left(X_{0}\right)$ +o $\left(t^{2}\right)$. Then $g(X)$ is also harmonic at $X_{0}$.

Without loss of generality, we assume $X_{0}$ is the origin. Then by Evans [2, Theorem 8, p. 109],

$$
g(X)-u(X)=k \log t+\sum_{m=1}^{\infty} \frac{1}{t^{m}}\left(a_{m} \cos m \theta+b_{m} \sin m \theta\right)
$$

where $u(X)$ is harmonic in $\bar{N}\left(0, t_{0}\right)$ and

$$
\frac{a_{m}}{t^{m}}=\frac{1}{\pi} \int_{0}^{2 \pi}[g(t \cos \theta, t \sin \theta)-u(t \cos \theta, t \sin \theta)] \cos m \theta d \theta
$$


for $m=1,2, \cdots$ and $0<t \leqq t_{0}$, with a similar formula for $b_{m} / t^{m}$.

Since $g(X)-u(X)$ is continuous on the punctured disc and in $L_{2}$ on the whole disc, we conclude that $a_{m}$ and $b_{m}$ are equal to zero for all $m$.

Furthermore, since $u_{1,0}(t)=u(0)+o\left(t^{2}\right)$, we conclude that $k=0$, and consequently that $g(X)$ is harmonic in $\bar{N}(0, t)$.

REMARK 1 . Let $F(X)$ be continuous in a domain $D$ and satisfy condition (5) at all points of $D$. Then by Radó [3, p. 14], $F(X)$ is harmonic in $D$.

5. Proof of theorem. Let us designate by $Z$ the set of discontinuity points of $F(X)$. Then $Z$ is dense-in-itself. For suppose that $X_{0}$ is an isolated point of $Z$. Then by Remark 1 and Lemma 3 there is an $N\left(X_{0}, t_{0}\right)$ such that $F(X)$ is harmonic in $\bar{N}\left(X_{0}, t_{0}\right)-X_{0}$. Furthermore by the Riesz-Fischer theorem, $F(X)$ is in $L_{2}$ on $\bar{N}\left(X_{0}, t_{0}\right)$. We consequently conclude from Lemmas 3 and 4 that $F(X)$ is harmonic and a fortiori continuous at $X_{0}$, which is a contradiction to the fact that $X_{0}$ is in $Z$.

Since $Z$ is dense in itself, $\bar{Z}$, the closure of $Z$, is a perfect set. But by (ii) of the theorem, $\bar{Z} \subset E$ is at most enumerable. But then by Saks [4, p. 55], $\bar{Z}$ and consequently $Z$ is null.

$F(X)$ is therefore harmonic in the whole plane and consequently $F(X)=F_{1, X}(t)$ for all $X$ and $t>0$. Therefore by Lemma 2

$$
F(X)=\frac{a_{0}}{4}\left[(x+y)^{2}+\frac{t^{2}}{2}\right]-2 \sum_{1 \leq|M|} a_{M} e^{i M X} \frac{J_{1}(|M| t)}{|M|^{3} t}
$$

Fixing $X$, we see that the series on the right side of (6) tends to zero as $t \rightarrow \infty$, and consequently that $a_{0}=0$ and $F(X)=0$. Therefore $\lim _{R \rightarrow \infty} \sum_{|M| \leqq R}\left(a_{M} /|M|^{2}\right) e^{i M X}=0$ for all $X$, and by the RieszFischer theorem $a_{M}=0$ for $|M| \neq 0$, which gives the theorem.

\section{Bibliography}

1. M. T. Cheng, Uniqueness of multiple trigonometric series, Ann. of Math. vol. 52 (1950) pp. 403-416.

2. G. C. Evans, The logarithmic potential, discontinuous Dirichlet and Neumann problems, Amer. Math. Soc. Colloquium Publications, vol. 6, New York, 1927.

3. T. Rad6, Subharmonic functions, Ergebnisse der Mathematik, vol. 5, no. 1, Berlin, 1937.

4. S. Saks, Theory of the integral, 2d. ed., Warsaw, 1937.

5. A. Zygmund, Trigonometrical series, Warsaw, 1935.

RUTGERS UNIVERSITY 